\title{
ACE Inhibitors or Sartans in the Treatment of Hypertension: A Needless Discussion?
}

\section{Jan Vaclavik ${ }^{1}$ and Jiri Sliva ${ }^{2^{\star}}$}

${ }^{1}$ Department of Internal Medicine, Palacky University, Olomouc, Czech Republic

${ }^{2}$ Department of Pharmacology, 2nd \& 3rd Faculty of Medicine, Charles University, Prague, Czech Republic

*Corresponding author: Jiri Sliva, Department of Pharmacology, 2nd \& 3rd Faculty of Medicine, Charles University, Prague, Czech Republic, Tel: 420737444823, Email: slivaj@seznam.cz

Received date: Oct 15, 2014, Accepted date: Dec 15, 2014, Published date: Dec 19, 2014

Copyright: ( 2014 Vaclavik J, et al. This is an open-access article distributed under the terms of the Creative Commons Attribution License, which permits unrestricted use, distribution, and reproduction in any medium, provided the original author and source are credited.

\begin{abstract}
ACE inhibitors and sartans are two broadly used groups of drugs in the treatment of essential arterial hypertension. Even though they possess similar efficacy, there has been a continuous discussion concerning their preferences. Hence, the authors consider the pros and cons regarding the efficacy of these two groups in the context of relevant published data.
\end{abstract}

Keywords: ACE inhibitors; Sartans; Raas; Hypertension; Adherence

\section{Commentry}

Most patients who suffer from essential arterial hypertension must be treated with a antihypertensive preparation in order to adequately control blood pressure. According to current ESH (European Society of Hypertension)/ESC (European Society of Cardiology) guidelines, physician have at their disposal five main groups of antihypertensives (as also referred to as basic antihypertensives) for use in either monotherapy or in combination: diuretics, beta-blockers (BB), calcium ion channel blockers (CaI), angiotensin convertase (ACE) inhibitors and sartans [1].

Basic antihypertensives possess very similar efficacies in terms of blood pressure reduction. When used at full dose, the average drop of systolic blood pressure (SBP) is $9.1 \mathrm{~mm} \mathrm{Hg}$ and diastolic blood pressure (DBP) $5.5 \mathrm{~mm} \mathrm{Hg}[1,2]$. However, before the initiation of pharmacotherapy, other co-morbidities (Table 1), contraindications and other factors must be taken into consideration. Considering the guidelines, initiation with renin-angiotensin-aldosterone system (RAAS) inhibitors i.e. ACE inhibitors or sartans is mostly recommended for a majority of co-morbidities. Alpha-1 blockers should only be considered in resistant hypertension, if no contraindication exists. Methyldopa (an alpha-2 agonist) should be preferred in hypertensive pregnant women.

\begin{tabular}{|c|c|c|c|c|}
\hline & $\begin{array}{l}\text { RAAS } \\
\text { inhibitors }\end{array}$ & Cal & diuretics & BB \\
\hline Left ventricle hypertrophy & $\mathrm{x}$ & $\mathrm{x}$ & & \\
\hline Metabolic syndrome & $\mathrm{x}$ & $\mathrm{x}$ & & \\
\hline Diabetes mellitus & $\mathrm{x}$ & & & \\
\hline Asymptomatic atherosclerosis & $\mathrm{x}$ & $\mathrm{x}$ & & \\
\hline Albuminuria/proteinuria & $\mathrm{x}$ & & & \\
\hline Renal dysfunction & $\mathrm{x}$ & & & \\
\hline
\end{tabular}

\begin{tabular}{|c|c|c|c|c|}
\hline Isolated systolic hypertension & & $\mathrm{x}$ & $\mathrm{x}$ & \\
\hline Atrial fibrillation & $\mathrm{x}$ & $\mathrm{x}$ & & $\mathrm{x}$ \\
\hline Previous myocardial infarction & $\mathrm{x}$ & & & $\mathrm{x}$ \\
\hline Angina pectoris & & $\mathrm{x}$ & & $\mathrm{x}$ \\
\hline Heart failure & $\mathrm{x}$ & & $\mathrm{x}$ & $\mathrm{x}$ \\
\hline
\end{tabular}

Table 1: Preferred groups of antihypertensives according to comorbidity (modified according to reference 1).

ACE inhibitors and sartans are equally effective in the reduction of blood pressure, proteinuria and impeding the progression of deteriorated renal functions [1,3,4]. Agents from both groups substantially reduce morbidity and mortality after myocardial infarction and heart failure [1,4]. Until recently, ACE inhibitors were recommended to be used in combination with sartans under some conditions (specifically, chronic kidney disease and proteinuria). Such a combination resulted in a slight decrease of SBP to approximately 4 $\mathrm{mmHg}$ [5] and a greater reduction of proteinuria when compared to monotherapy which was implemented in small-scale studies [6]. However, subsequent large studies evaluating the effect of this combination on mortality did not decrease the occurrence of cardiovascular death, myocardial infarction, stroke or heart failure when compared to monotherapy. Moreover, the use of combination was associated with more common hypotension, diarrhea, and renal insufficiency [7]. Hence, recently adopted guidelines by ESH/ESC for the treatment of essential arterial hypertension recommended to avoid concomitant administration of ACE inhibitors and sartans in hypertonic patients [1].

Several meta-analyses have been performed in recent years. However, their results have become the source of discussion regarding the potential superiority of ACE inhibitors in the reduction of allcause and cardiovascular mortality over sartans. One of the most controversial is the meta-analysis conducted by van Vark et al, which comprised of 20 clinical trials (following the responses of nearly 159,000 patients) [8]. The authors demonstrated that RAAS inhibitors 
reduced total mortality and cardiovascular mortality by $5 \%$ ( $0.032)$ and $7 \%(p=0.018)$, respectively, when compared to controls (placebo or other groups of antihypertensives). Secondary analyses showed a significant decrease in total mortality after ACE inhibitors $(-10 \% ; p=0.004)$, while the effect of sartans remained statistically non-significant $(-1 \% ; \mathrm{p}=0.683)$. The difference in the influence of ACE inhibitors and sartans on cardiovascular mortality was nonsignificant $(\mathrm{p}=0.227)[8]$.

Upon closer examination of the data, the difference between ACE inhibitors and sartans might be easily explained by the heterogenity of the compared population. Over a decade ago, it was shown that the risk of cardiovascular mortality is doubled with each $20 / 10 \mathrm{~mm} \mathrm{Hg}$ increase of blood pressure. Additionally, the average decrease of systolic blood pressure by $2 \mathrm{~mm} \mathrm{Hg}$ results in $7 \%$ decrease of risk of death from ischaemic heart disease or other vascular causes and $10 \%$ decrease of risk of stroke death [9]. Hence, it is not surprising that the most substantial decrease in mortality was observed in those studies involved in the meta-analysis, where the highest value of baseline blood pressure in the studied population was present and where the most extensive decrease of blood pressure after treatment was observed (data extracted from the LIFE, HYVET, ASCOT-BPLA trials). On the other hand, the decrease of the baseline blood pressure was less impressive in the majority of studies with sartans, in some of them the initial blood pressure was even normal (data obtained from JIKEI HEART, HIJ-CREATE, NAVIGATOR trials). The heterogeneity of the involved trials is additionally supported by the comparable cardiovascular mortality in studies with ACE inhibitors in the active treatment arm (9.1/1000 patient/year) to control groups in studies with sartans (9.2/1000 patient/year) [8]. Importantly, the authors of this paper emphasize that both groups of RAAS inhibitors are difficult to compare objectively based on the available data. Hence, the results of the meta-analysis must be interpreted very carefully. In addition, the authors of these studies concur, that a change in current clinical praxis is not necessary [8].

Another commonly discussed meta-analysis by Savarese et al. compared ACE inhibitors and sartans in 26 randomized control trials which consisted of more than 100,000 study subjects who had not previously experienced heart failure [10]. Both ACE inhibitors and sartans administration were associated with a significant decrease of the composite endpoint (myocardial infarction, stroke and cardiovascular death), stroke and newly diagnosed diabetes mellitus [10]. However, the extent of decreased myocardial infarction and total mortality was significant with the use of ACE inhibitors. Interestingly, according to the authors, the principal limitation of this work lies in the fact that the involved studies with ACE inhibitors (performed earlier) was comprised of patients with coronary artery disease or other manifest cardiovascular disease, while studies with sartans (performed later) was mostly comprised of diabetic patients or patients diagnosed with impaired glucose tolerance [10]. Thus, also in this case, ACE inhibitors and sartans, cannot be adequately compared [10].

The most robust and important randomized clinical trial comparing the efficacy of the ACE inhibitor, ramipril with the sartan (telmisartan) was the ONTARGET study conducted in 2008 [7]. It involved more than 25,000 patients with manifest cardiovascular disease or diabetic patients at high risk (more than two thirds of them suffered from hypertension). There was no significant difference observed between the influences of ramipril and telmisartan on the frequency of myocardial infarction, stroke, hospitalization due to heart failure, cardiovascular mortality or total mortality [7]. The only difference between these two approaches was a higher occurrence of adverse effects (dry cough) and more frequent discontinuation of treatment in patients who were prescribed ramipril [7].

Instead of a discussion about the superiority of one group over another, the focus should be on the different pharmacologic properties of each particular substance. This approach might be feasible especially because the above mentioned discussion presumes the classeffect of all substances involved in ACE inhibitors and the same in sartans. However, there are distinct patterns in pharmacokinetics (rate of absorption, biologic half-life, route of elimination, lipophilicity, tissue binding...) and pharmacodynamics (affinities to target structures, trough to peak ratio...). Only some substances have data from clinical trials that describe their beneficial effects on major cardiovascular events (MACE). Both ramipril and perindopril administered in the HOPE and EUROPA studies, respectively, showed a significant reduction of manifest cardiovascular disease, while quinapril and trandolapril prescribed to patients in the QUIET and PEACE studies did not have a similar effect even though the extent of reduced blood pressure was very similar. Only the ONTARGET study provided data demonstrating the role of telmisartan in cardiovascular prevention $[11,12]$ while the rest of the sartans have proven to be beneficial (CHARM trials etc.) in patients with heart failure or left ventricle hypertrophy.

In the context of the aforementioned data on the efficacy of the two major groups of RAAS inhibitors, it should be mentioned that a very recent meta-analysis which compared ACE inhibitors and sartans in diabetic patients, in the context of total mortality, cardiovascular mortality and MACE was performed [13]. In total, 23 studies with ACE inhibitors $(n=32,827)$ and 13 studies with sartans $(n=23,867)$ were analyzed. ACE inhibitors significantly reduced total mortality by $13 \%$, however, no similar influence of sartans was observed to controls when compared. It is important to note that, there is an apparent difference in the total number of evaluated subjects, heterogenity in length of follow-up and distinct basic characteristics of studied populations. For example, the patients who used ACE inhibitors more commonly suffered from coronary artery or other vascular disease. Hence, a more apparent effect of the treatment might be expected. Even in this paper, the authors also concluded, that the theoretical superiority of ACE inhibitors over sartans in diabetic patients cannot clearly be established.

Last but not least, the full dose of any antihypertensive drug, i.e. the dose which has well-documented effects noted in clinical trials, must be administered in order to achieve the maximal prevention of cardiovascular events. A lower dose will not likely be as effective - for example $2.5 \mathrm{mg}$ ramipril prescribed once daily decelerates the progression of atherosclerosis in carotid arteries at a slower rate than $10 \mathrm{mg}$ of ramipril 10 given once per day [14].

Of importance, the use of an adequate dose of a RAAS inhibitor to adequately influence the cardiovascular risk does not only depend on the optimum prescribed dose, but also the risk is affected by adherence and persistency to the treatment. Sartans owns a better safety profile when compared to ACE inhibitors, hence a better compliance can be extrapolated. This theoretical background is supported by a population-based Italian study (a cohort of 445,356 patients) performed by Corrao et al. interestingly sartans is mentioned as a group with the best compliance when compared to the other groups of antihypertensives including $\mathrm{ACE}$ inhibitors, $\mathrm{CaI}, \mathrm{BB}$, and diuretics [13]. The better compliance may be additionally achieved by use of 
Citation: Vaclavik J, Sliva J (2014) ACE Inhibitors or Sartans in the Treatment of Hypertension: A Needless Discussion?. J Hypertens 3: 191. doi:10.4172/2167-1095.1000191

Page 3 of 3

fixed combinations of antihypertensives enabling either synergism (RAAS inhibitors + diuretics etc.) or decreased risk of adverse effects (RAAS inhibitors + CaI etc.) or both (RAAS inhibitors + CaI) [14].

In conclusion, both ACE inhibitors and sartans are first line antihypertensives used in a vast majority of patients. According to ESH/ESC guidelines, these groups are equally effective in the treatment of hypertension. Definitely, they have beneficial effects in hypertonic patients in terms of a decreased risk in cardiovascular events, however, differences regarding drug efficacy between individual drugs due to their distinct pharmacologic properties is highly likely. The superiority of one group of RAAS inhibitors over another has not been distinctly shown in numerous trials.

\section{References}

1. Mancia G, Fagard R, Narkiewicz K, et al. (2013) 2013 ESH/ESC Guidelines for the management of arterial hypertension: the Task Force for the management of arterial hypertension of the European Society of Hypertension (ESH) and of the European Society of Cardiology (ESC). J Hypertens 31: 1281-1357.

2. Law MR, Wald NJ, Morris JK, Jordan RE (2003) Value of low dose combination treatment with blood pressure lowering drugs: analysis of 354 randomised trials. BMJ 326: 1427.

3. Heran BS, Wong MM, Heran IK, Wright JM (2008) Blood pressure lowering efficacy of angiotensin receptor blockers for primary hypertension. Cochrane Database Syst Rev 4:CD003822.

4. Burnier M, Vuignier Y, Wuerzner G (2014) State-of-the-art treatment of hypertension: established and new drugs. Eur Heart J 35: 557-562.

5. Doulton TW, He FJ, MacGregor GA (2005) Systematic review of combined angiotensin-converting enzyme inhibition and angiotensin receptor blockade in hypertension. Hypertension 45: 880-886.

6. Kunz R, Friedrich C, Wolbers M, Mann JF (2008) Meta-analysis: effect of monotherapy and combination therapy with inhibitors of the renin angiotensin system on proteinuria in renal disease. Ann Intern Med 148: 30-48.

7. ONTARGET Investigators, Yusuf S, Teo KK, Pogue J (2008) Telmisartan, ramipril, or both in patients at high risk for vascular events. N Engl J Med 358:1547-1559.

8. van Vark LC, Bertrand M, Akkerhuis KM, (2012) Angiotensinconverting enzyme inhibitors reduce mortality in hypertension: a metaanalysis of randomized clinical trials of renin-angiotensin-aldosterone system inhibitors involving 158,998 patients. Eur Heart J 33: 2088-2097.

9. Lewington S, Clarke R, Qizilbash N, Peto R, Collins R; Prospective Studies Collaboration (2002) Age-specific relevance of usual blood pressure to vascular mortality: a meta-analysis of individual data for one million adults in 61 prospective studies. Lancet 360: 1903-1913.

10. Savarese G, Costanzo P, Cleland JG, Vassallo E, Ruggiero D, et al. (2013) A meta-analysis reporting effects of angiotensin-converting enzyme inhibitors and angiotensin receptor blockers in patients without heart failure. J Am Coll Cardiol 61: 131-142.

11. Cheng J, Zhang W, Zhang X (2014) Effect of angiotensin-converting enzyme inhibitors and angiotensin II receptor blockers on all-cause mortality, cardiovascular deaths, and cardiovascular events in patients with diabetes mellitus: a meta-analysis. JAMA Intern Med 174: 773-785.

12. Lonn E, Yusuf S, Dzavik V (2001) Effects of ramipril and vitamin E on atherosclerosis: the study to evaluate carotid ultrasound changes in patients treated with ramipril and vitamin E (SECURE). Circulation 103: 919-925.

13. Corrao G, Zambon A, Parodi A, Poluzzi E, Baldi I, et al. (2008) Discontinuation of and changes in drug therapy for hypertension among newly-treated patients: a population-based study in Italy. J Hypertens 26 : 819-824.

14. Corrao G, Nicotra F, Parodi A, Zambon A, Heiman F, et al. (2011) Cardiovascular protection by initial and subsequent combination of antihypertensive drugs in daily life practice. Hypertension 58: 566-572. 